\title{
Zugang zu urbanen Grünflächen im Kontext von Hitzeereignissen am Beispiel von Jena
}

\author{
Anika Zorn ${ }^{1}$ Susann Schäfer ${ }^{1}$ Uwe Kurmutz² Sophie Köhler ${ }^{1}$ \\ Eingegangen: 23. Februar 2021 / Überarbeitet: 4. Mai 2021 / Angenommen: 6. Mai 2021 / Online publiziert: 22. Juni 2021 \\ (c) Der/die Autor(en) 2021
}

\section{Zusammenfassung}

Der Klimawandel und die bauliche Beschaffenheit von Städten führen zu zunehmenden Hitzeereignissen in Städten, die Auswirkungen auf Gesundheit und Wohlbefinden der Bewohner*innen haben können. Urbane Grünflächen werden als stadtplanerische Möglichkeit betrachtet, das Mikroklima innerhalb der Stadt zu beeinflussen und das Wohlbefinden der Bewohner*innen zu erhöhen. Bisherige Studien zeigen jedoch, dass diese Form der stadtplanerischen Klimaanpassung Fragen der sozialen Gerechtigkeit aufwirft: Nicht alle Bewohner*innen der Städte haben gleichen Zugang zu kühlenden Grünflächen. Ausgehend von der Frage, welche räumlichen Strategien vulnerable Gruppen bei Hitzeereignissen verfolgen, wird in diesem Beitrag analysiert, inwiefern es soziale oder räumliche Unterschiede im Zugang zu urbanen Grünflächen gibt. Als Fallbeispiel dient hier die Stadt Jena (Thüringen). Datengrundlage stellt eine systematische, teilstandardisierte Befragung der Bewohner*innen der besonders hitzebelasteten Stadtteile aus dem Jahr 2018 dar. In dieser Studie wird dezidiert die Perspektive, das Verhalten und die Bedarfe der Stadtbewohner*innen erhoben. Die Ergebnisse legen nahe, dass ältere Personen an heißen Tagen eher in ihrer Wohnung bzw. ihrem Haus bleiben und öffentliche Räume meiden. Dieser Rückzug kann zum sozialen Ausschluss dieser Bevölkerungsgruppe führen, wenn nicht stadtplanerisch reagiert wird. Kühlende Grünflächen sind zwar in Jena prinzipiell zahlreich vorhanden - allerdings ungleich verteilt, was sich am Nutzungsverhalten der Befragten widerspiegelt. Der von vulnerablen sozialen Gruppen bewohnte Norden der Stadt ist weitgehend abgeschottet von Grünflächen, die den Bedarfen der Befragten entsprechen. Der Beitrag spricht Handlungsempfehlungen für die Stadtentwicklung aus und beleuchtet, wie die Stadt Jena auf die Studie reagiert hat.

Schlüsselwörter Umweltgerechtigkeit · Klimaanpassung $\cdot$ Hitzeinsel $\cdot$ Stadtplanung $\cdot$ Klimawandel $\cdot$ Vulnerabilität

Anika Zorn

anika.zorn@uni-jena.de

1 Institut für Geographie, Friedrich-Schiller-Universität Jena, Löbdergraben 32, 07743 Jena, Deutschland

2 Thüringer Institut für Nachhaltigkeit und Klimaschutz, Leutragraben 1, 07743 Jena, Deutschland 


\begin{abstract}
Climate change and the built-up environment of cities lead to increasing heat events in cities, which can have an impact on the health and well-being of the inhabitants. Public green spaces are regarded as an urban planning opportunity to influence the microclimate within the city and increase the well-being of residents. However, previous studies show that this form of urban climate adaptation raises issues of social justice: not all city residents have equal access to cooling green spaces. Based on the question of which spatial strategies vulnerable groups pursue during heat events, this paper analyzes to what extent there are social or spatial differences in access to urban green spaces. The city of Jena (Thuringia) serves as a case study. The data are based on a systematic, partially standardized survey of the residents of the most heat-stressed parts of the city from 2018. In this study, the perspective, the behavior, and the needs of the city's residents are determined. The results suggest that older people are more likely to stay in their apartment or house and avoid public spaces on hot days. This withdrawal can lead to social exclusion of this population group if urban planning does not react. Cooling green spaces are in principle plentiful in Jena-but unevenly distributed, which is reflected in the usage behavior of the respondents. The north of the city, where vulnerable social groups live, is largely isolated from green spaces that meet the needs of the respondents. The article proffers guidance on urban development and sheds light on how the city of Jena has reacted to the study.
\end{abstract}

Keywords Environmental justice $\cdot$ Climate change adaptation $\cdot$ Heat island $\cdot$ Urban planning $\cdot$ Climate change Vulnerability

\section{Einleitung}

Der Klimawandel ist eine große Herausforderung für Städte (Bulkeley 2012). Insbesondere Hitzewellen können zu einer kritischen Belastung für die Stadtbevölkerung werden, da Hitze die menschliche Gesundheit negativ beeinflusst und die Sterblichkeit ansteigt (Patz et al. 2005; Kovats und Hajat 2008). Dies gilt vor allem für vulnerable Gruppen wie Senior*innen, Kinder oder Menschen mit bestimmten Vorerkrankungen. Städtische Grünflächen oder auch urbane Klimaoasen (Parks, Wälder, Gründächer, Bäche und Gemeinschaftsgärten) werden aufgrund ihrer kühlenden Funktion als geeignet angesehen, negative gesundheitliche Auswirkungen zu mildern (Bowler et al. 2010; Lee und Maheswaran 2011). Neben positiven Effekten auf das städtische Mikroklima haben mehrere Studien gezeigt, dass diese Räume positiv zur physischen und psychischen Gesundheit der Stadtbewohner*innen beitragen, soziale Begegnungen ermöglichen und somit die Lebensqualität der Stadtbevölkerung erhöhen (Gozalo et al. 2018; Thompson et al. 2012; Vargas-Hernandez et al. 2017). Aufgrund ihrer positiven Eigenschaften sind diese Elemente zu einem kritischen Aspekt der Governance geworden (Sturiale und Scuderi 2019) - insbesondere in städtischen Ballungsräumen, in denen das Potenzial von Hitzeinseln stark ist. Empirische Studien haben gezeigt, dass urbane Grünflächen in US-amerikanischen und chinesischen Städten ungleichmäBig verteilt und überwiegend für bestimmte Gemeinschaften zugänglich sind (Heynen, Perkins und Roy 2006) - angesichts häufiger auftretenden Hitzewellen aufgrund des Klimawandels ist die Frage nach städtischen Grünflächen zu einer Frage der Gerechtigkeit in Bezug auf den Zugang zu und die Governance dieser Flächen geworden (Kabisch und Haase 2014; Rosol 2010).

Der vorliegende Beitrag greift diese Problematik auf und befasst sich mit der Frage nach dem Umgang mit Hitzeereignissen unter besonderer Berücksichtigung der städtischen Grünflächen und deren Zugang durch die Stadtbevölkerung, wobei ein systematischer Ansatz verfolgt wird, bei dem die gesamte Bevölkerung des am stärksten von sommerlicher Hitzebelastung betroffenen Stadtgebiets in Jena (Thüringen) befragt wird. Im Gegensatz zu anderen Studien über städtische Grünflächen berücksichtigt diese Studie systematisch die Perspektive der betroffenen Stadtbevölkerung, analysiert Verhalten bei Hitze sowie die Nutzung, den Zugang und die Eigenschaften von städtischen Grünflächen und entwickelt Handlungsempfehlungen für die Stadtentwicklung.

\section{Klimaanpassung in Jena}

Die Universitätsstadt Jena liegt im mittleren Saaletal, im Klimabereich der südostdeutschen Becken und Hügel (TLUBN o.J.). Das lokale Klima wird durch die kesselartige Lage beeinflusst und weist ein milderes Klima als die umgebenden Hochflächen auf, verbunden mit einem nur schwachen Luftaustausch und potenziellem Wärmestau (Böer und Schmidt 1970; Hoffmann et al. 2014). Die mittlere jährliche Lufttemperatur liegt bei $9,9^{\circ} \mathrm{C}$, die Jahresniederschlagssumme bei $610 \mathrm{~mm}$; es gibt 54 Sommertage (Höchsttemperatur $\mathrm{T}_{\max } \geq 25^{\circ} \mathrm{C}$ ) und 13 Heiße Tage $\left(\mathrm{T}_{\max } \geq 30^{\circ} \mathrm{C}\right.$ ) pro Jahr (DWD-Klimastation Jena, Klimaperiode 1981 bis 2010). Hier können Anstiege der Jahresmitteltemperatur um 1,2 Kelvin (K) sowie der Sommer- 
und Heißen Tage um 9,8 bzw. 3,5 seit der Klimaperiode 1901-1930 verzeichnet werden, die dem anthropogen induzierten Klimawandel zuzurechnen sind (Kurmutz 2017). Jena weist einen städtischen Wärmeinseleffekt mit einem Jahresmittel von etwa $1 \mathrm{~K}$ und Maxima von bis zu 4-7K am frühen Morgen während autochthoner Wetterlagen auf (Dörfer 2004; Hoffmann et al. 2014). Aktuelle Klimaprojektionen für die RCP-Szenarien lassen eine signifikante Verstärkung der Hitzebelastung in Jena in den kommenden Dekaden erwarten (Kurmutz 2017; TLUBN 2020).

Aufgrund der Hitzewellen von 2003 und 2006 rückte die Klimafolgenanpassung in Jena ab 2008 in den Fokus der Stadtverwaltung. Im Jahr 2009 erfolgte eine erste Kurzstudie zum Thema, die den Ausgangspunkt für die Jenaer Klimaanpassungsstrategie bildete (JenKAS, 2009 bis 2012), einem gesamtstädtischen Konzept, welches die Themen Wärmebelastung, Trockenstress, Starkregenerosion und Hochwasser-/Starkregenüberflutungen betrachtete. Die während des JenKAS-Prozesses etablierte Arbeitsgruppe aus Verwaltung, Wissenschaft und Landesbehörden setzt ihre Arbeit bis heute fort. Hier entstehen Projektideen für vertiefende thematische Teilkonzepte, die innerhalb der Gesamtstrategie nicht ausführlich untersucht werden konnten oder bestehende Inhalte aktualisieren. Beispiele sind das Stadtbaumkonzept (2014-2016), eine Untersuchung der Wärmebelastungen an Kindertagesstätten und Grundschulen (2017) sowie jüngst ein Projekt zu grünen Klimaoasen im urbanen Stadtraum (2017-2020). Aktuell befinden sich ein Hitzeaktionsplan sowie eine Aktualisierung der Stadtklimaanalyse aus JenKAS in Bearbeitung.

Grünräume in ihren diversen Erscheinungen spielen in Jena eine wichtige Rolle und tragen wesentlich zum Stadtbild bei. Jena wird in Ost und West von Wald auf den Hochflächen eingerahmt. Im Saaletal dominiert die Saaleaue mit großen Parkanlagen. Zudem bieten zahlreiche weitere Grünräume wie Freibäder, Friedhöfe, der Botanische Garten, Grünflächen etc. bioklimatische Entlastung während sommerlicher Hitzeperioden. Neben der Bioklimaregulation stellen diese Grünräume weitere relevante Ökosystemleistungen bereit, u. a. Regenwasserdrainage und Überschwemmungsregulierung, Kohlenstoffspeicherung, Luftfilterung, Habitate mit Trittsteinwirkung, Raum für soziale Begegnung sowie Sport und Spiel. Die Sicherung und Erschließung qualitätsvoller städtischer Grünflächen ist unter der Zielvorgabe einer doppelten Innenentwicklung somit ebenso notwendig wie die Schaffung neuer Wohnund Gewerbeflächen (Kurmutz et al. 2020).

\section{Systematische Befragung der Bürger*innen}

Es wurde eine systematische Befragung durchgeführt, um zu ermitteln, wie sich die Bewohner*innen der Stadt Jena bei Hitzeereignissen verhalten und welche Rolle Grünanlagen dabei spielen. Die Befragung zu grünen Klimaoasen wurde mithilfe eines teilstandardisierten Fragebogens durchgeführt, der ein vielfältiges Spektrum verschiedener Fragenarten enthielt. Die Erhebung der Daten umfasste eine persönliche, schriftliche Haushaltsbefragung im innerstädtischen, hitzebelasteten Jenaer Untersuchungsgebiet (mehr als 10 Heiße Tage $\left[\mathrm{T}_{\max } \geq 30^{\circ} \mathrm{C}\right]$ im Jahresmittel der Klimaperiode 1971-2000) sowie eine Online-Bürger*innenbefragung. Der Befragungszeitraum belief sich auf insgesamt vier Wochen im Juni 2018. Das Untersuchungsgebiet erstreckt sich nördlich der Saale zwischen den Stadtteilen Jena-West, Jena-Zentrum, Jena-Nord und Zwätzen. Die Haushalte wurden innerhalb des Untersuchungsgebiets mit einer Zufallsstichprobe ausgewählt, um die Validität und Repräsentativität der Ergebnisse gewährleisten zu können. Die ausgefüllten Fragebögen wurden persönlich abgeholt. Dabei übertraf die Rücklaufquote von $73 \%$ bei Weitem die Erwartungen, und deutete auf ein hohes stadtpolitisches Mitbestimmungsinteresse der Anwohner*innen hin. Die Online-Befragung erreichte 595 Menschen, von denen 183 den Fragebogen vollständig ausfüllten, woraus sich eine Rücklaufquote von ca. $31 \%$ errechnete. Jene Online-Umfragen, die sich aufgrund der abgefragten Postleitzahl eindeutig dem Untersuchungsgebiet zuzuordnen ließen, ergaben gemeinsam mit der Haushaltsbefragung einen Datensatz mit einem $N$ von 243; die übrigen 132 Befragungen wurden der Kontrollgruppe zugeordnet. Fehlerhafte und unvollständige Fragebögen wurden aussortiert. Die Digitalisierung und Auswertung der Daten erfolge mithilfe der Statistiksoftware IBM SPSS Statistics for Windows 24, Armonk, New York, USA (Döring und Bortz 2016).

\section{Urbane Grünflächen in Jena}

\section{Relevanz und Betroffenheit von Hitze}

Fast drei Viertel der Befragten gaben an, dass sie die Sommer in den letzten Jahren wärmer bzw. heißer wahrnehmen. Nur etwa jeder Fünfte empfand, dass sich an den Sommern nichts geändert habe, wie Abb. 1 zu entnehmen ist. Auffällig ist, dass $65 \%$ der Befragten, die keine Veränderung wahrnehmen, unter 34 Jahren alt ist. Dass es heißer geworden ist, empfinden überwiegend Personen zwischen 35 und 54 Jahren. Heiße Sommertage haben für circa ein Drittel der Befragten einen leicht negativen Einfluss und für $22 \%$ sogar einen sehr negativen Einfluss auf ihr Wohlbefinden. Etwa $15 \%$ der Bürger*innen fühlen sich jedoch von der sommerlichen Hitze positiv beeinflusst. Die Antwortverteilung ist in Abb. 2 dargestellt. Auch beim Wohlbefinden ist ein leichter Zusammenhang mit dem Alter festzustellen: 
Abb. 1 Wahrgenommene Veränderung der Temperaturen im Sommer
Anteile der Antworten auf die Frage: "Haben Sie den Eindruck, dass die Sommer in Jena in den vergangenen Jahren heißer geworden sind?" $(\mathrm{N}=221)$

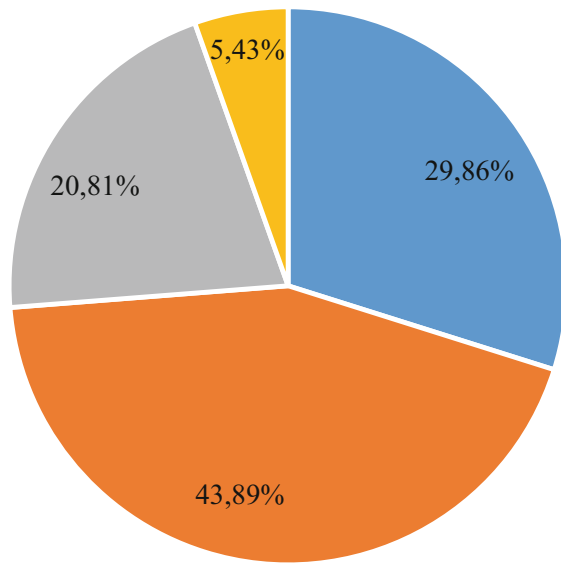

- Ja, es ist heißer geworden $\quad$ Ja, es ist wärmer geworden $\quad$ Nein, es hat sich nichts verändert $\quad$ Sonstiges
Abb. 2 Einfluss der Heißen Tage auf das körperliche Wohlbefinden

\begin{abstract}
Sommertage (mehr als $30^{\circ} \mathrm{C}$ Tageshöchsttemperatur) auf Ihr körperliches
Wohlbefinden?" ( $\mathrm{N}=232)$

Anteile der Antworten auf die Frage: "Welchen Einfluss haben heiße
\end{abstract}

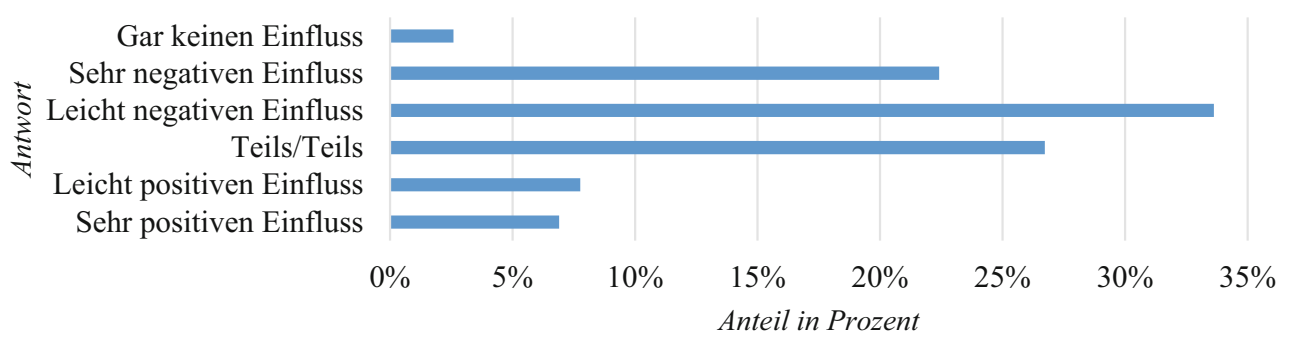

Das Wohlbefinden bei höheren Temperaturen sinkt mit dem Alter. Die Strategien im Umgang mit der stärker wahrgenommenen Hitzebelastung variieren ebenfalls mit dem Alter. Die Mehrheit der Personen ab 35 Jahren verbringt mehr Zeit in ihrer Wohnung bzw. ihrem Haus, wobei der Anteil dieser Personen mit steigendem Alter zunimmt. Das gleiche Muster zeigt sich bei Personen, die längere Aufenthalte an bestimmten Orten in der Stadt vermeiden. Knapp 50\% der Bürger*innen gaben zudem an, dass sie besonders stark besonnte Wege umgehen bzw. umfahren.

\section{Bedeutung und Nutzung von städtischen Grün- und Parkanlagen}

Knapp $98 \%$ der Befragten gaben an, dass ihnen Grün- und Parkanlagen in Jena wichtig bis sehr wichtig seien, wohingegen niemand innerstädtische Grünflächen als weniger wichtig oder gar unwichtig bewertete. Die Hälfte der Befragten empfindet die vorhandenen Grünflächen als viel zu wenig bzw. zu wenig. Über $38 \%$ der befragten Bewohner*innen sind mit der Anzahl vorhandener Flächen zu- frieden, während nur $12 \%$ diese als zu viel einstufen. Die Ergebnisse sind in Abb. 3 visualisiert. Auffällig ist, dass die Zufriedenheit mit der Grünflächensituation der Stadt nicht mit dem Vorhandensein eines eigenen Gartens oder dem Einkommen zusammenhängt. Ein Großteil der Befragten ( $83 \%$ bzw. $70 \%$ ) nutzen die Grün- und Parkflächen zum Ausruhen und Erholen bzw. um die Natur zu genießen. Über $70 \%$ der Befragten nutzen die Anlagen als Treffpunkt mit Bekannten und Freund*innen, mehr als die Hälfte spaziert dort. Circa ein Drittel der Bürger*innen grillen bzw. picknicken in den Grünanlagen, treiben Sport oder baden in nahegelegenen Gewässern. Etwa ein Viertel nutzt die Anlagen zum Spielen mit Kindern. Die wichtigsten Aspekte an den genutzten Grün- und Parkanlagen sind Schatten durch Bäume bzw. Bepflanzungen durch Bäume und Büsche, Rasenflächen, die Nähe zum Wasser und zum Wohnort. Zu den essenziellen gewünschten Ausstattungsmerkmalen zählen darüber hinaus Mülleimer und Bänke. Etwa zwei Drittel der Befragten möchten die Grünanlagen zu Fuß bzw. mit dem Fahrrad erreichen können, etwa ein Drittel mit dem öffentlichen Personennahverkehr. 
Anteile der Antworten auf die Frage: "Wie bewerten Sie die Anzahl der bestehenden Grün- und Parkflächen in Jena?" (N=230)

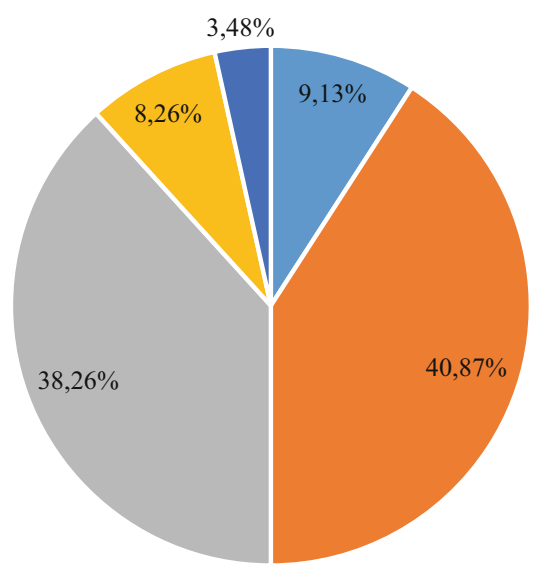

घ Viel zu gering $\quad$ Zu wenig $\quad$ Ausreichend $\quad$ Zu hoch $\quad$ Viel zu hoch

Abb. 3 Bewertung der Anzahl der bestehenden Grün- und Parkflächen in Jena

\section{Bevorzugte und gemiedene Aufenthaltsorte}

Die meist frequentierten Grünanlagen sind Abb. $4 \mathrm{zu}$ entnehmen. Über drei Viertel der Befragten gaben den großen Paradies-Park als bevorzugte Grünfläche im Sommer an. Weitere wichtige Aufenthaltsorte sind die Ufer der Saale oder andere wassernahe Orte (Camsdorfer/Wenigenjenaer Ufer, Landfeste, Lommerweg an der Leutra). Städtische Gärten (Prinzessinnengarten bzw. Botanischer Garten) sind ebenfalls beliebte Ziele im Sommer.

Die räumliche Verteilung der genutzten Grünflächen macht deutlich, dass sich die besuchten Grünanlagen vorwiegend auf das Zentrum der Stadt inklusive Paradies-Park konzentrieren. Zudem werden Wälder außerhalb der Stadt aufgesucht - allerdings nur von circa $8 \%$ der Befragten. Es ist auffällig, dass im nördlichen Teil des Untersuchungsgebietes kaum Grünflächen vorliegen, die frequentiert werden.

\section{Zusammenfassung und Interpretation}

Ein Großteil der Bevölkerung in Jena nimmt steigende Sommertemperaturen wahr, was sich bei den meisten negativ auf das Wohlbefinden auswirkt. Vor allem ältere Menschen, die als hitzevulnerabel gelten, fühlen sich bei zunehmenden Temperaturen unwohl. Die Bürger*innen entwickeln eigene Strategien, um diesem Unwohlsein in ihrem Alltag entgegenzuwirken: Es wird ersichtlich, dass sich Personen mit zunehmendem Alter an heißen Tagen eher in private Räume zurückziehen und den öffentlichen Raum meiden. Bei der Zunahme von Hitzeperioden im Zuge des
Klimawandels könnte dies ohne Anpassungsmaßnahmen vonseiten der Stadt dazu führen, dass ältere Personen den öffentlichen Raum an heißen Tagen immer weniger nutzen. Aus diesem Grund ist die Berücksichtigung vulnerabler Gruppen in der städtischen Klimaanpassung besonders hervorzuheben. Zu der Entwicklung von urbanen Grünflächen zählt dabei aber auch die Planung der sozialen Infrastruktur unter Berücksichtigung klimatischer Veränderungen, z. B. Kindertagesstätten, Krankenhäuser, Senior*innenheime.

Vor dem Hintergrund der zunehmend wahrgenommenen Hitzebelastung spielen für fast alle Bürger*innen Grünanlagen eine bedeutende Rolle. Diese werden im Sommer für Erholungszwecke genutzt. Kühlende Attribute wie schattenspendende Bäume oder Zugang zum Wasser sind dafür von besonderer Relevanz. Die Grünanlagen weisen zudem eindeutig eine soziale Funktion auf: Sie werden als Treffpunkte genutzt. Gestützt wird dieser Fakt davon, dass selbst Bürger*innen in Besitz eines Gartens, welcher für sie eine ähnliche Erholungs- und Kühlfunktion erbringen könnte, öffentliche Grünanlagen nicht weniger nutzen. Der Zugang zu diesen Orten soll einfach und für die meisten Befragten ohne Auto möglich sein.

Obwohl Jena weitgehend von Wäldern umschlossen ist und somit viele Bewohner*innen potenziell einen Zugang zu Grünflächen haben, werden die Waldgebiete nur von wenigen im Sommer aufgesucht. Eine Ursache hierfür ist das steile Relief der Jena umgebenden Talhänge, das besonders unter sommerlichen Hitzebedingungen einer einfachen Nutzung der Wälder auf den Hochflächen entgegensteht und näher gelegene innerstädtische Grünflächen in den Fokus der Befragten rückt, die die gewünschte Ausruhfunktion besser erfüllen. Im Norden der Stadt existiert nur ein Friedhof als größere Grünanlage, der jedoch nur von wenigen als Grünfläche im Sommer genutzt wird - vermutlich, weil er ähnlich wie die umliegenden Wälder nicht der Ausruhfunktion entspricht, aber auch Sport oder der Verzehr von Mahlzeiten verboten sind. Somit gibt es im nördlichen Teil des hitzebetroffenen Untersuchungsgebietes kaum fußläufig erreichbare Grünanlagen, die den Bedarfen der Bürger*innen entsprechen. Es konnte aus den erhobenen Daten kein $\mathrm{Zu}$ sammenhang zwischen Einkommen und der Zufriedenheit mit der Anzahl der Grünflächen festgestellt werden. Dennoch lässt sich mit Blick auf soziodemografische Merkmale des Bezirks Jena-Nord feststellen, dass dort 2017 die höchste Arbeitslosigkeit des Untersuchungsgebiets (8,6\%) gemessen wurde. Im Bezirk Nord II wohnen besonders viele ältere Menschen: Der Altenquotient von Nord II war im Erhebungsjahr mit 69 der höchste aller Bezirke Jenas (Jena o.J.). Die Abgeschiedenheit von Grünflächen im hitzebetroffenen Jenaer Norden betrifft folglich vulnerable soziale Gruppen. Dort bestand also Handlungsbedarf - auf den die Stadt Jena in der Zwischenzeit reagiert hat. So wurde in Folge der Befragung im Jahr 2020 im nördlichen Teil 
Abb. 4 Lage des Untersuchungsgebietes (Gebiete mit einer mittleren jährlichen Anzahl Heißer Tage über 10 in der Klimaperiode 1971-2000) im Verhältnis zu Einrichtungen besonders hitzesensitiver Personengruppen und bevorzugten Grünanlagen; Markergröße entsprechend der Gesamtzahl der Nennungen (Quellen: DWD 2012, Stadt Jena 2018, eigene Erhebungen 2018)

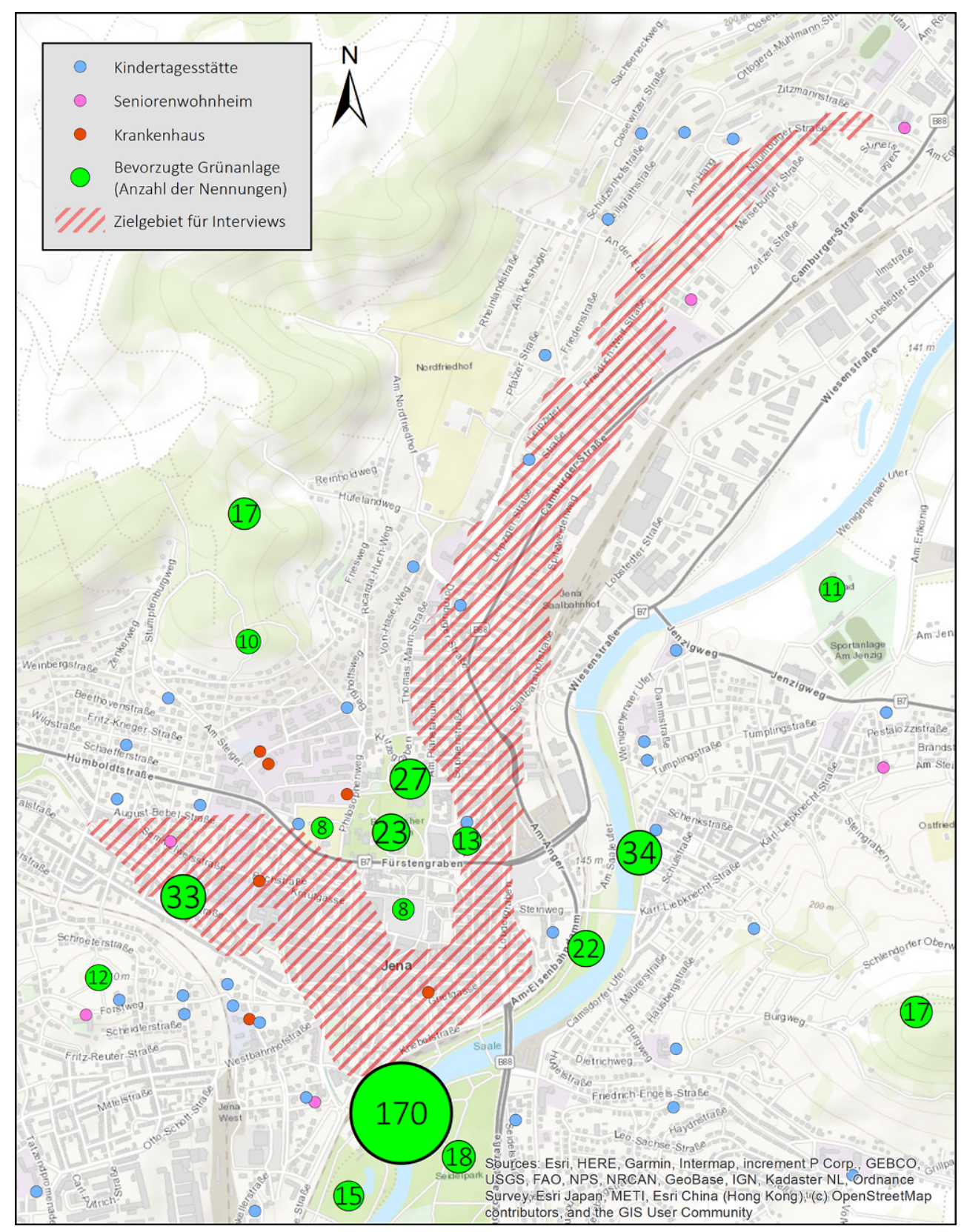

der Stadt eine weitere Grünfläche ausgewiesen und entwickelt (siehe Abb. 5); eine weitere Grünfläche im Norden erhält eine Aufwertung. Zudem hat sich die Stadt zum Ziel gemacht, bestehende Klimaoasen zu erhalten, aufzuwerten und in Zukunft noch neue Grünflächen zu schaffen.

\section{Fazit und Handlungsempfehlungen}

Mit zunehmendem Alter werden Hitzeereignisse als unangenehm empfunden, was dazu führt, dass ältere Menschen Innenstädte meiden und währenddessen in den eigenen vier Wänden verweilen. Dies kann im Zuge des Klima- wandels zu temporärer sozialer Exklusion dieser Bevölkerungsgruppe führen. Eine klimagerechte Stadtentwicklung muss beschattete Wege und kühlende Grünflächen bereitstellen, um präventiv diesem Ausschluss entgegenzuwirken. Bei der Planung von Senior*inneneinrichtungen und anderen Einrichtungen, die hitzevulnerablen Gruppen gewidmet sind, gilt dies besonders zu beachten. Grünanlagen sind für nahezu alle Befragten gern genutzte kühlende Orte mit einer starken sozialen Funktion. Aus den Ergebnissen der Studie lässt sich erkennen, dass sich ein Großteil der Stadtbewohner*innen noch mehr Grünanlagen wünscht. Vor allem das von vulnerablen sozialen Gruppen bewohnte nördliche Stadtgebiet weist einen Mangel an geeigneten 


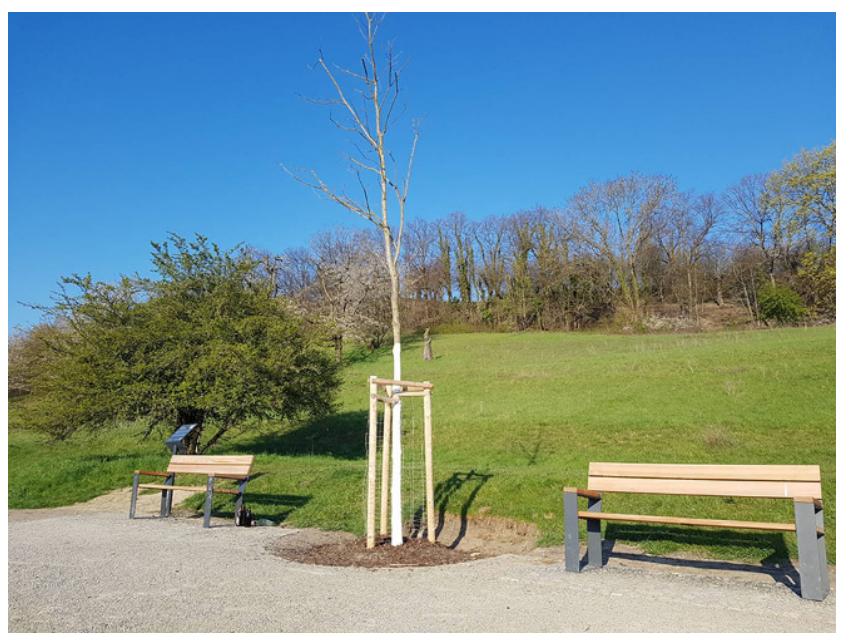

Abb. 5 Am Heiligenberg im Jenaer Norden wurde eine neue Grünfläche von der Stadt geschaffen. (Foto: Susann Schäfer 2021)

Klimaoasen auf, auf den die Stadt Jena bereits reagiert. Für eine bürger*innenorientierte Stadtentwicklung ist es demnach empfehlenswert, die Bedarfe und das Nutzungsverhalten der Stadtbewohner*innen unter besonderer Berücksichtigung vulnerabler Gruppen zu erfassen, um Stadtteile mit wenigen (genutzten) Grünflächen zu identifizieren und dort durch Schaffung neuer oder Aufwertung bestehender Flächen einen gerechten Zugang zu kühlenden Grünflächen zu ermöglichen.

Danksagung Wir bedanken uns bei der Stadt Jena für die Finanzierung der Erhebung. Besonders hilfreich waren die Anmerkungen von zwei anonymen Reviewer*innen. Für diesen Input bedanken wir uns herzlich. Außerdem bedanken wir uns bei Sophie Tzschabran für die Unterstützung bei der Verfassung des Beitrages.

Funding Open Access funding enabled and organized by Projekt DEAL.

Open Access Dieser Artikel wird unter der Creative Commons Namensnennung 4.0 International Lizenz veröffentlicht, welche die Nutzung, Vervielfältigung, Bearbeitung, Verbreitung und Wiedergabe in jeglichem Medium und Format erlaubt, sofern Sie den/die ursprünglichen Autor(en) und die Quelle ordnungsgemäß nennen, einen Link zur Creative Commons Lizenz beifügen und angeben, ob Änderungen vorgenommen wurden.

Die in diesem Artikel enthaltenen Bilder und sonstiges Drittmaterial unterliegen ebenfalls der genannten Creative Commons Lizenz, sofern sich aus der Abbildungslegende nichts anderes ergibt. Sofern das betreffende Material nicht unter der genannten Creative Commons Lizenz steht und die betreffende Handlung nicht nach gesetzlichen Vorschriften erlaubt ist, ist für die oben aufgeführten Weiterverwendungen des Materials die Einwilligung des jeweiligen Rechteinhabers einzuholen.

Weitere Details zur Lizenz entnehmen Sie bitte der Lizenzinformation auf http://creativecommons.org/licenses/by/4.0/deed.de.

\section{Literatur}

Böer W, Schmidt G (1970) Klimagebiete und bioklimatische Situation der Sanatorien, Kur- und Erholungsorte. In: Atlas der Deutschen
Demokratischen Republik. Hermann-Haack-Verlag, Gotha, Leipzig (Blatt 9)

Bowler DE, Buyung-Ali L, Knight TM, Pullin AS (2010) Urban greening to cool towns and cities: a systematic review of the empirical evidence. Landsc Urban Plan 97:147-155

Bulkeley H (2012) Cities and climate change. Routledge critical introductions to urbanism and the city. Taylor \& Francis, London

Dörfer S (2004) Stadtklimatische Untersuchungen im Raum Jena. Diplomarbeit, Jena

Döring N, Bortz J (2016) Forschungsmethoden und Evaluation in den Sozial- und Humanwissenschaften. Springer, Berlin Heidelberg

Gozalo GR, Morillas JMB, Gonzalez DM, Moraga PA (2018) Relationships among satisfaction, noise perception, and use of urban green spaces. Sci Total Environ 624:438-450

Heynen N, Perkins HA, Roy P (2006) The political ecology of uneven urban green space-The impact of political economy on race and ethnicity in producing environmental inequality in Milwaukee. Urban Aff Rev 42:3-25

Hoffmann K, Bivour W, Früh B, Koßmann M, Voß P-H (2014) Klimauntersuchungen in Jena für die Anpassung an den Klimawandel und seine erwarteten Folgen. Ein Ergebnisbericht. https:// www.dwd.de/DE/klimaumwelt/klimaforschung/klimawirk/ stadtpl/stadtklimaprojekte/projekt_jena/stadtpl_jena_node.html. Zugegriffen: 4. Febr. 2021 (Deutscher Wetterdienst, Deutsche Meteorologische Bibliothek)

Jena (o.J.) Bezirksstatistik. https://statistiken.jena.de/ stadtbezirksstatistik.html. Zugegriffen: 8. Apr. 2021

Kabisch N, Haase D (2014) Green justice or just green? Provision of urban green spaces in Berlin, Germany. Landsc Urban Plan 122:129-139

Kovats RS, Hajat S (2008) Heat stress and public health: a critical review. Annu Rev Public Health 29:41-55

Kurmutz U (2017) Analyse der lokalen Auswirkungen des Klimawandels und Ableitung von Planungshinweisen für die Klimaanpassung am Beispiel der Stadt Jena. https://www.db-thueringen.de/ receive/dbt_mods_00032639. Zugegriffen: 4. Febr. 2021 (Dissertation, Jena)

Kurmutz U, Gebhardt O, Schwamberger A, Zander S, Knetsch S, Mann M, Müller A (2020) Grüne Klimaoasen im urbanen Stadtraum Jenas. Abschlussbericht zum BBSR-Forschungsprojekt Green Urban Labs (im Erscheinen)

Lee A, Maheswaran R (2011) The health benefits of urban green spaces: a review of the evidence. J Public Health 33:212-222

Patz JA, Campbell-Lendrum D, Holloway T, Foley JA (2005) Impact of regional climate change on human health. Nature 438:310-317

Rosol M (2010) Public participation in post-Fordist urban green space governance: the case of community gardens in Berlin. Int J Urban Reg Res 34:548-563

Sturiale L, Scuderi A (2019) The role of green infrastructures in urban planning for climate change adaptation. Climate 7(10):119

Thompson CW, Roe J, Aspinall P, Mitchell R, Clow A, Miller D (2012) More green space is linked to less stress in deprived communities: evidence from salivary cortisol patterns. Landsc Urban Plan 105:221-229

Thüringer Landesamt für Umwelt, Bergbau und Naturschutz TLUBN (2020) Zukünftige Entwicklung des Klimas in Thüringen. https:// tlubn.thueringen.de/klima/zukuenftiges-klima. Zugegriffen: 4. Febr. 2021

Thüringer Landesamt für Umwelt, Bergbau und Naturschutz TLUBN (o. J.) Die vier Thüringer Klimabereiche und ihre klimacharakteristischen Merkmale. http://www.tlug-jena.de/uw_raum/ umweltregional/thueringen/09_klimabereiche.html. Zugegriffen: 4. Febr. 2021

Vargas-Hernandez JG, Pallagst K, Zdunek-Wielgolaska J (2017) Urbans green spaces as a component of an ecosystem. Functions, services, users, community, involvement, initiatives and actions. Rev Urban 37:1-26 